\title{
Efficacy and Safety of Alirocumab in Patients with Heterozygous Familial Hypercholesterolemia not Adequately Controlled with Current Lipid-Lowering Therapy: Design and Rationale of the ODYSSEY FH Studies
}

\author{
John J. P. Kastelein • Jennifer G. Robinson • Michel Farnier • Michel Krempf • \\ Gisle Langslet • Christelle Lorenzato • Daniel A. Gipe • Marie T. Baccara-Dinet
}

Published online: 20 May 2014

(C) The Author(s) 2014. This article is published with open access at Springerlink.com

\begin{abstract}
Background Individuals with heterozygous familial hypercholesterolemia (heFH) have higher levels of low-density lipoprotein cholesterol (LDL-C) and are predisposed to premature cardiovascular disease. Alirocumab is a fully-human, monoclonal antibody targeted to proprotein convertase subtilisin/kexin type 9 currently in Phase 3 development for the treatment of hypercholesterolemia. Described here are three ODYSSEY Phase 3 trials, FH I (NCT01623115), FH II (NCT01709500) and HIGH FH (patients with heFH and LDL-C levels $\geq 160 \mathrm{mg} / \mathrm{dL}$ ) (NCT01617655), in which alirocumab is further evaluated in the heFH population.
\end{abstract}

\author{
J. J. P. Kastelein $(\bowtie)$ \\ Department of Vascular Medicine, Academic Medical Center, \\ University of Amsterdam, Meibergdreef 9, Room F4-159.2, 1105 \\ AZ Amsterdam, The Netherlands \\ e-mail: j.j.kastelein@amc.uva.nl \\ J. G. Robinson \\ University of Iowa, Iowa City, IA, USA \\ M. Farnier \\ Point Medical, Dijon, France \\ M. Krempf \\ CHU de Nantes - Hopital Nord Laennec, Saint-Herblain, France \\ G. Langslet \\ Lipid Clinic, Oslo University Hospital, Oslo, Norway \\ C. Lorenzato \\ Sanofi, Paris, France \\ D. A. Gipe \\ Regeneron Pharmaceuticals, Inc., Tarrytown, NY, USA \\ M. T. Baccara-Dinet \\ Sanofi, Montpellier, France
}

Methods Multicenter, multinational, randomized, doubleblind, placebo-controlled studies have been designed to evaluate efficacy and safety of alirocumab in more than 800 patients with heFH who are not adequately controlled with a maximally-tolerated stable daily dose of statin for $\geq 4$ weeks prior to the screening visit, with or without other lipidlowering therapy. Patients are randomized $(2: 1)$ to receive alirocumab or placebo via a $1-\mathrm{mL}$ subcutaneous autoinjection every 2 weeks (Q2W) for 78 weeks. In studies FH I and II, if their Week 8 LDL-C level is $\geq 70 \mathrm{mg} / \mathrm{dL}$, patients will undergo a dose uptitration from 75 to $150 \mathrm{mg}$ alirocumab Q2W at Week 12. In HIGH FH, patients will receive alirocumab $150 \mathrm{mg}$ Q2W throughout the entire treatment period. The primary efficacy endpoint in all three studies is the percent change in calculated LDL-C from baseline to Week 24.

Conclusions The ODYSSEY FH studies are three Phase 3 studies aiming to further evaluate the efficacy and long-term safety of alirocumab as an effective therapeutic option for patients with heFH.

Keywords Alirocumab $\cdot$ Heterozygous familial hypercholesterolemia $\cdot$ LDL-C $\cdot$ PCSK9

\section{Introduction}

Heterozygous familial hypercholesterolemia (heFH) is a hereditary lipid metabolism disorder that predisposes affected individuals to cardiovascular (CV) disease [1]. Patients with heFH typically have very high low-density lipoprotein cholesterol (LDL-C) levels - often $>190 \mathrm{mg} / \mathrm{dL}$ at the time of diagnosis - that are associated with high risk for premature CV disease [2, 3]. However, a recent consensus statement 
highlights that in most countries less than $1 \%$ of patients with heFH are, in fact, diagnosed [4].

Findings from observational studies have shown that the risk of coronary heart disease (CHD) is reduced in heFH patients receiving statin therapy [5-7]; however, even with this treatment, the risk of CHD is still greater in heFH patients than in the general population [5]. Despite the availability of lipid-lowering therapy (LLT), approximately $80 \%$ of patients with heFH do not reach the recommended levels of LDL-C [8-12]. Given the increased CV risk in the heFH population, there is a need to provide patients with additional and more intensive lipid-lowering therapy $[4,13]$.

Proprotein convertase subtilisin/kexin type 9 (PCSK9), a therapeutic target currently under investigation, binds to LDL$\mathrm{C}$ receptors, resulting in their degradation so that fewer receptors are available on liver cells to remove excess LDL-C from the plasma [14-17].

PCSK9 inhibition has the potential to provide a complementary mechanism to other LLTs to significantly reduce LDL-C beyond the efficacy of statins [15, 17-25]. Alirocumab (formerly REGN727/SAR236553; SanofiRegeneron) is a fully human, monoclonal antibody targeted to PCSK9 currently in Phase 3 development for the treatment of hypercholesterolemia.

In Phase 2 trials, alirocumab demonstrated significant reductions in LDL-C levels in patients receiving concomitant statin or statin plus ezetimibe therapy [NCT01288443; NCT01266876; NCT01288469; 21, 23, 24]. In these studies, alirocumab significantly $(p<0.001)$ reduced mean LDL-C levels by up to $72.4 \%$, and also showed favorable trends in other atherogenic lipid parameters including lipoprotein(a) [Lp(a)], triglycerides, non-high-density lipoprotein cholesterol (non-HDL-C), apolipoprotein (Apo) B, and anti-atherogenic fractions HDL-C and Apo A1. Specifically, a Phase 2 trial demonstrated that alirocumab reduced mean LDL-C levels by up to $67.9 \%(p<0.0001)$ in patients with heFH [24]. No doselimiting adverse events (AEs) were identified in the Phase 2 studies [21, 23, 24], with the most common treatmentemergent AE (TEAE) in patients receiving alirocumab being mild injection site reaction. Five serious AEs (SAEs) occurred in four patients $(1.5 \%)$ who received alirocumab and two SAEs occurred in two patients $(2.6 \%)$ who received placebo.

The ODYSSEY Phase 3 alirocumab clinical trial program is designed to further assess the efficacy and safety of alirocumab in a range of clinical settings. The trials within the program evaluate a treat-to-goal approach, using a flexible dosing strategy for individualized therapy based on degree of LDL-C lowering required to achieve an adequate treatment response. This approach is designed to address patient populations unable to achieve desired LDL-C levels with the current standard of care. The program comprises a total of 14 studies planned to include more than 23,500 patients in over 2,000 study centers worldwide. Of these studies, the ODYSSEY program also includes a large cardiovascular outcomes trial evaluating the long-term impact of alirocumab and lower levels of LDL-C on the occurrence of cardiovascular events in 18,000 patients after a recent $(<52$ weeks) acute coronary syndrome event, with a randomized treatment period of 64 months. The aim of the ODYSSEY FH studies is to assess the efficacy and safety of alirocumab in patients with heFH who, despite their maximally-tolerated statin dose, with or without other LLT, continue to have suboptimal LDL-C levels and require additional pharmacologic management.

\section{Methods}

\section{Study Design}

All three Phase 3 ODYSSEY FH clinical trials are multicenter, multinational, randomized, double-blind, placebo-controlled studies designed to evaluate the efficacy and safety of alirocumab in patients with heFH not adequately controlled with their current LLT.

The FH I study (ClinicalTrials.gov identifier: NCT01623115) is being conducted at 89 sites across North America, Europe and South Africa, and has a planned population of 471 patients. The FH II study (ClinicalTrials.gov identifier: NCT01709500) is being conducted at 26 sites across Europe and has a planned population of 250 patients.

Both the FH I and II studies enroll heFH patients who have LDL-C levels $\geq 70 \mathrm{mg} / \mathrm{dL}$ at the screening visit and have a history of documented CVD, or heFH patients with LDL-C $\geq 100 \mathrm{mg} / \mathrm{dL}$ at the screening visit without a history of documented CVD (Fig. 1).

The HIGH FH study (ClinicalTrials.gov identifier: NCT01617655) is being conducted at 33 sites across North America, Europe and South Africa, and has a planned population of 105 patients with heFH and LDL-C levels $\geq 160 \mathrm{mg} / \mathrm{dL}$ at the screening visit (Fig. 1).

The studies are being performed in accordance with the ethical principles that have their origin in the Declaration of Helsinki and all applicable amendments laid down by the World Medical Assemblies and the International Conference Harmonisation guidelines for Good Clinical Practice. Institutional review board or independent ethics committee approval of the protocols and informed consent forms were obtained from each study site, and written informed consent was obtained from all patients.

\section{Study Objectives}

The primary efficacy objective in all three studies is to demonstrate the reduction of LDL-C by alirocumab, in comparison with placebo, as add-on therapy to stable, maximally 
Fig. 1 Study designs. a ODYSSEY FH I, b ODYSSEY FH II and $\mathbf{c}$ ODYSSEY HIGH FH. $\mathrm{CV}$ cardiovascular, $h e F H$ heterozygous familial hypercholesterolemia, $L D L-C$ lowdensity lipoprotein cholesterol, $L L T$ lipid-lowering therapy, NCEP ATP III TLC National Cholesterol Education Program Adult Treatment Panel III Therapeutic Lifestyle Changes, $Q 2 W$ every 2 weeks, $R$ randomization, $S C$ subcutaneous. ${ }^{a}$ At the end of the double-blind treatment period, patients will be offered the possibility to enter an open-label extension study, in which they will receive alirocumab. If patients opt out of entering the open-label treatment period, they will enter the 8-week follow-up period a

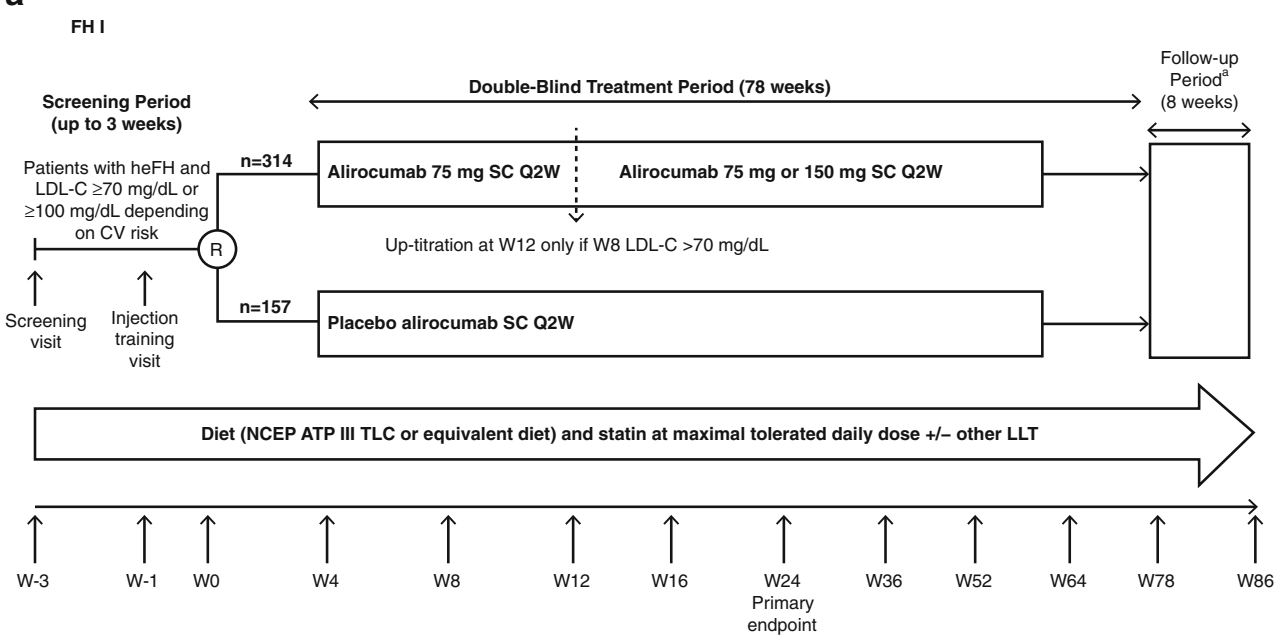

b

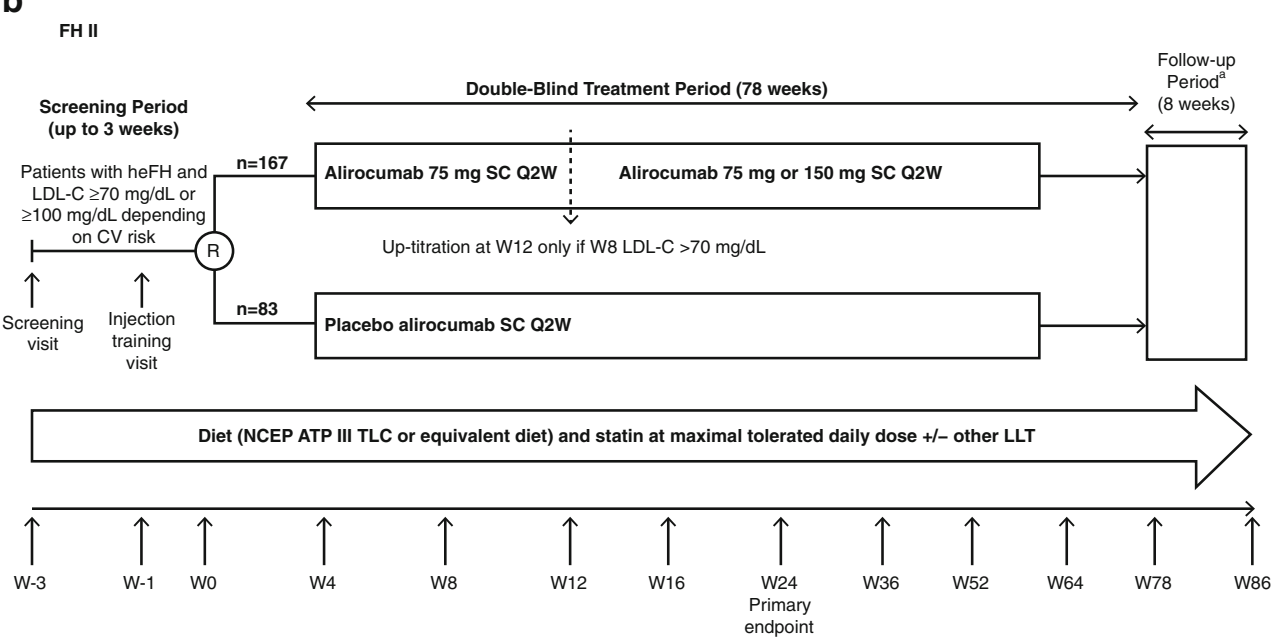

C

HIGH FH

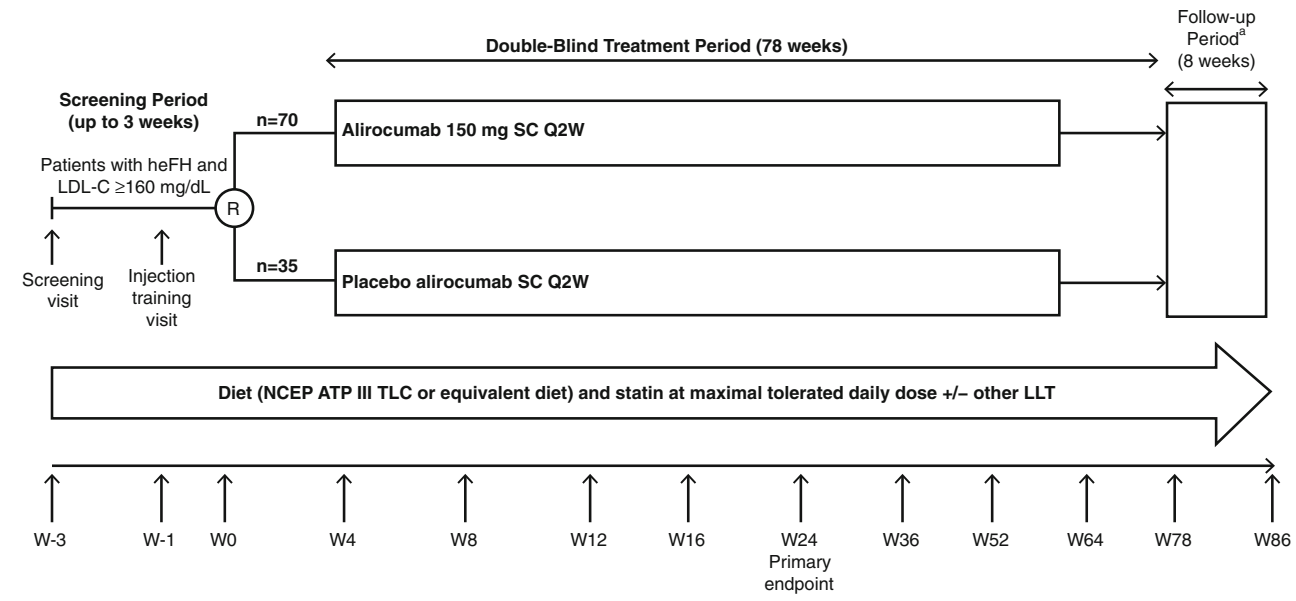

tolerated daily statin therapy, with or without other LLT, after 24 weeks of treatment in patients with heFH. Secondary objectives common to all three studies include the evaluation of the effect of alirocumab in comparison with placebo on LDL-C after 12 weeks of treatment; the effect of alirocumab on other lipid parameters, e.g., Apo B, non-HDL-C, total cholesterol, Lp(a), HDL-C, triglycerides and Apo A1 levels; the long-term effect of alirocumab on LDL-C; the safety and tolerability of alirocumab; and evaluation of the development of anti-alirocumab antibodies. 


\section{Inclusion Criteria}

The principal inclusion criteria for all three studies are patients with heFH who are not adequately controlled with a maximally tolerated stable daily dose of statin for at least 4 weeks prior to the screening visit, with or without other LLT (Table 1).

The diagnosis of heFH must be made either by genotyping or clinical criteria. For those patients not genotyped, the clinical diagnosis may be based on either the Simon Broome criteria [26], meeting the criteria for definite FH, or the World Health Organization (WHO)/Dutch Lipid Network criteria [27] with a score of $>8$ points.

\section{Exclusion Criteria}

The principal exclusion criteria for the FH I and II studies include patients with different LDL-C thresholds depending on their cardiovascular risk status: LDL-C $<70 \mathrm{mg} / \mathrm{dL}$ $(<1.81 \mathrm{mmol} / \mathrm{L})$ in patients with history of documented CVD and LDL-C $<100 \mathrm{mg} / \mathrm{dL}(<2.59 \mathrm{mmol} / \mathrm{L})$ in patients without history of documented CVD.

The principal exclusion criterion for the HIGH FH study includes patients with an LDL-C level of $<160 \mathrm{mg} / \mathrm{dL}$ $(<4.14 \mathrm{mmol} / \mathrm{L})$ at the screening visit (Table 1$)$. Other key exclusion criteria common to the three studies are shown in Table 1.
Study Procedures

The three FH studies consist of three periods: a screening period of up to 3 weeks, during which the patient or another designated person is trained to self-inject/inject study medication $(1 \mathrm{~mL}$ administered via auto-injector); a 78-week double-blind treatment period; and an 8-week off-treatment follow-up period. It should be noted that, at the end of the 78-week treatment period in each study, patients will be offered the possibility to enter an open-label extension study, in which they will receive alirocumab. If patients opt out of entering the open-label treatment period, they will enter the 8-week follow-up period.

In FH I, FH II and HIGH FH, all eligible patients are randomized $2: 1$ to alirocumab or placebo. Randomization is stratified according to history of myocardial infarction (MI) or ischemic stroke for balance across study arms, intensity of statin treatment (atorvastatin 40 to $80 \mathrm{mg}$ daily, or rosuvastatin 20 to $40 \mathrm{mg}$ daily versus simvastatin irrespective of the daily dose, atorvastatin below $40 \mathrm{mg}$ daily or rosuvastatin below $20 \mathrm{mg}$ daily), and geographic region (where applicable).

At randomization, treatment kit numbers are allocated using a centralized treatment allocation system, which is either an interactive voice response system or an interactive web response system, depending on the site preference. Study patients, principal investigators and study site personnel remain blinded to all randomization assignments throughout the

Table 1 Principal inclusion and exclusion criteria for ODYSSEY FH I, FH II, and HIGH FH

\footnotetext{
Principal inclusion criteria for all three studies

Patients with heFH who are not adequately controlled ${ }^{\mathrm{a}}$ with a maximally-tolerated stable daily dose of statin $^{\mathrm{b}}$ for at least 4 weeks prior to the screening visit, with or without other LLT

Principal exclusion criteria

Not on a stable dose of LLT (including statin) for at least 4 weeks and/or fenofibrate for at least 6 weeks, as applicable, prior to the screening visit or from screening to randomization

Currently taking a statin that is not simvastatin, atorvastatin or rosuvastatin taken daily at a registered dose

Receiving daily doses above atorvastatin $80 \mathrm{mg}$, rosuvastatin $40 \mathrm{mg}$ or simvastatin $40 \mathrm{mg}$ (except for patients on simvastatin $80 \mathrm{mg}$ for more than 1 year, who are eligible)

Use of fibrates, other than fenofibrate, within 6 weeks of the screening visit

Fasting serum triglycerides $>400 \mathrm{mg} / \mathrm{dL}$ ( $>4.52 \mathrm{mmol} / \mathrm{L}$ ) at the screening visit

Known history of homozygous FH

FH I and II

History of documented CVD and LDL-C $<70 \mathrm{mg} / \mathrm{dL}(<1.81 \mathrm{mmol} / \mathrm{L})$ at the screening visit

Without history of documented CVD and LDL-C $<100 \mathrm{mg} / \mathrm{dL}(<2.59 \mathrm{mmol} / \mathrm{L})$ at the screening visit

\section{HIGH FH}

LDL-C $<160 \mathrm{mg} / \mathrm{dL}(<4.14 \mathrm{mmol} / \mathrm{L})$ at the screening visit AND patient only on statin monotherapy without additional LLT

$C V D$ cardiovascular disease, $F H$ familial hypercholesterolemia, heFH heterozygous familial hypercholesterolemia, $L D L-C$ low-density lipoprotein cholesterol, $L L T$ lipid-lowering therapy, $M I$ myocardial infarction

${ }^{a}$ FH I and II: not adequately controlled defined as an LDL-C $\geq 70 \mathrm{mg} / \mathrm{dL}$ at the screening visit in patients with a history of documented CVD OR LDL-C $\geq 100 \mathrm{mg} / \mathrm{dL}$ at the screening visit in patients without a history of documented CVD. HIGH FH: not adequately controlled defined as an LDL-C $\geq 160 \mathrm{mg} /$ $\mathrm{dL}$ at the screening visit

${ }^{\mathrm{b}}$ Maximally-tolerated statin dose defined as: the highest tolerable registered dose of daily statin currently administered to the patient, that is rosuvastatin $20 \mathrm{mg}$ or $40 \mathrm{mg}$ daily; atorvastatin $40 \mathrm{mg}$ or $80 \mathrm{mg}$ daily; simvastatin $80 \mathrm{mg}$ daily (if already on this dose for $>1$ year). Patients who are not able to be on any of the above statin doses should be treated with the dose of daily atorvastatin, rosuvastatin, or simvastatin which is considered appropriate for the patient, according to the investigator's judgment
} 
study. All lipid and anti-drug antibody results collected after randomization are masked.

In the three studies, the first auto-injection during the double-blind treatment period takes place at the site on the day of randomization (Week 0). Subsequent auto-injections are done by the patient or another designated person such as a spouse or relative at a patient-preferred location and occur every 2 weeks.

In FH I and FH II, patients randomized to alirocumab will receive a $75 \mathrm{mg} 1 \mathrm{~mL}$ dose every 2 weeks (Q2W). Patients randomized to placebo will receive a $1 \mathrm{~mL}$ subcutaneous placebo injection in an identical auto-injector to maintain blinding. At Week 12, patients randomized to alirocumab undergo a dose uptitration to $150 \mathrm{mg}$ Q2W, also given as a $1 \mathrm{~mL}$ auto-injection, if the Week $8 \mathrm{LDLC}$ level is $\geq 70 \mathrm{mg} / \mathrm{dL}$ $(1.81 \mathrm{mmol} / \mathrm{L})$. The continuation of the $75 \mathrm{mg}$ dose or dose up-titration to the $150 \mathrm{mg}$ dose will occur in an automated process without site or patient awareness.

In HIGH FH, at study entry, patients have an LDL-C level of $\geq 160 \mathrm{mg} / \mathrm{dL}$ ( $4.14 \mathrm{mmol} / \mathrm{L})$ and they will be randomized to receive a $150-\mathrm{mg} 1 \mathrm{~mL}$ dose of alirocumab or placeboalirocumab, without the uptitration process, from randomization to the end of the 78-week double-blind treatment period.

During the three FH studies, all patients are asked to follow a stable, National Cholesterol Education Program Adult Treatment Panel III Therapeutic Lifestyle Changes diet or equivalent throughout the entire study duration from screening to completion.

\section{Key Study Endpoints and Assessments}

The primary efficacy endpoint in all three studies is the percent change in calculated LDL-C from baseline to Week 24 , using all LDL-C values regardless of adherence to treatment (intent-to-treat [ITT] approach). Key secondary endpoints are summarized in Table 2.

In FH I, II and HIGH FH, LDL-C will be calculated using the Friedewald formula at screening and at all time points during the double-blind treatment periods. In FH I and II, if triglycerides exceed $400 \mathrm{mg} / \mathrm{dL}(4.52 \mathrm{mmol} / \mathrm{L})$ then the central laboratory will reflexively measure LDL-C (via the beta quantification method) rather than calculating it. LDL-C will also be measured (via the beta quantification method) at Week 0 and Week 24.

On-site patient assessments take place at Weeks $0,12,24$, $36,52,64,78$, and 86 , the end-of-study visit. All laboratory tests are performed by the central laboratory.

Safety parameters (AEs [including adjudicated CV events categorized as CHD death, non-fatal MI, fatal and non-fatal ischemic stroke, unstable angina requiring hospitalization, congestive heart failure requiring hospitalization], laboratory data [blood biochemistry, hematology and urinalysis], vital signs and electrocardiogram) are assessed throughout the study.
Table 2 Primary and key secondary endpoints common to FH I, FH II and HIGH FH

Primary endpoint

$\%$ change in calculated LDL-C from baseline to Week 24 in the ITT population, using all LDL-C values regardless of adherence to treatment (ITT estimand)

Key secondary endpoints

$\%$ change in calculated LDL-C from baseline to Week 24 in the mITT population, using all LDL-C values during the efficacy treatment period (on-treatment estimand)

$\%$ change in LDL-C from baseline to Week 12 (ITT estimand)

$\%$ change in LDL-C from baseline to Week 12 (on-treatment estimand)

$\%$ change in Apo B from baseline to Week 24 (ITT estimand)

$\%$ change in Apo B from baseline to Week 24 (on-treatment estimand)

$\%$ change in non-HDL-C from baseline to Week 24 (ITT estimand)

$\%$ change in non-HDL-C from baseline to Week 24 (on-treatment estimand)

$\%$ change in total cholesterol from baseline to Week 24 (ITT estimand)

$\%$ change in Apo B from baseline to Week 12 (ITT estimand)

$\%$ change in non-HDL-C from baseline to Week 12 (ITT estimand)

$\%$ change in total cholesterol from baseline to Week 12 (ITT estimand)

$\%$ change in calculated LDL-C from baseline to Week 52 (ITT estimand)

Proportion of very high $\mathrm{CV}$ risk patients reaching calculated LDL-C $<70 \mathrm{mg} / \mathrm{dL}(1.81 \mathrm{mmol} / \mathrm{L})$ or high CV risk patients reaching calculated LDL-C $<100 \mathrm{mg} / \mathrm{dL}(2.59 \mathrm{mmol} / \mathrm{L})$ at Week 24 (ITT estimand)

Proportion of very high CV risk patients reaching calculated LDL-C $<$ $70 \mathrm{mg} / \mathrm{dL}(1.81 \mathrm{mmol} / \mathrm{L})$ or high CV risk patients reaching calculated LDL-C $<100 \mathrm{mg} / \mathrm{dL}(2.59 \mathrm{mmol} / \mathrm{L}$ ) at Week 24 (ontreatment estimand)

Proportion of patients reaching LDL-C $<70 \mathrm{mg} / \mathrm{dl}(1.81 \mathrm{mmol} / \mathrm{L})$ at Week 24 (ITT estimand) ${ }^{\mathrm{a}}$

Proportion of patients reaching LDL-C $<70 \mathrm{mg} / \mathrm{dl}(1.81 \mathrm{mmol} / \mathrm{L})$ at Week 24 (on-treatment estimand) ${ }^{\mathrm{a}}$

$\%$ change in Lp(a) from baseline to Week 24 (ITT estimand)

$\%$ change in HDL-C from baseline to Week 24 (ITT estimand)

$\%$ change in fasting TG from baseline to Week 24 (ITT estimand)

$\%$ change in Apo A1 from baseline to Week 24 (ITT estimand)

$\%$ change in Lp(a) from baseline to Week 12 (ITT estimand)

$\%$ change in HDL-C from baseline to Week 12 (ITT estimand)

$\%$ change in fasting TG from baseline to Week 12 (ITT estimand)

$\%$ change in Apo A1 from baseline to Week 12 (ITT estimand)

Apo apolipoprotein, $F H$ familial hypercholesterolemia, $H D L-C$ high-density lipoprotein cholesterol, ITT intent-to-treat, $L D L$ - $C$ low-density lipoprotein cholesterol, $L p(a)$ lipoprotein(a), $m I T T$ modified ITT, $T G$ triglycerides

${ }^{\text {a }}$ Proportion of patients reaching LDL-C $<70 \mathrm{mg} / \mathrm{dL}$ (ITT and on-treatment estimand) are the last key secondary endpoints for HIGH FH

Statistical Design and Analysis

Sample Size Determination

In each study (FH I, FH II and HIGH FH), a total sample size of 45 patients ( 30 in the alirocumab and 15 in the placebo 
group) will have $95 \%$ power to detect a difference in mean percent change in LDL-C of $30 \%$ with a 0.05 two-sided significance level, assuming a common standard deviation of $25 \%$ and all these 45 patients having an evaluable primary endpoint.

However, to meet regulatory requirements across the program, sample sizes were increased to assess the safety of alirocumab appropriately. Therefore, the final total sample sizes were increased and rounded to 471 in FH I, 250 in FH II and 105 in HIGH FH.

\section{Analysis Populations for Each of the FH Studies}

Primary Analysis The primary analysis population will be the ITT population and will comprise all randomized patients with at least one baseline calculated LDL-C value available and at least one calculated LDL-C value available between Weeks 4 and 24 (regardless of treatment adherence). The percent change from baseline in LDL-C at Week 24 will be analyzed using a mixed effect model with repeated measures (MMRM) approach. All available post-baseline data from Week 4 to 24 (on- and off-treatment) will be used, in which missing data will be accounted for by the MMRM $[28,29]$. The model includes fixed categorical effects of treatment group, randomization strata time point, treatment-by-time point and strata-by-time point interaction as well as the continuous fixed covariates of baseline LDL-C value and baseline value-by-time point interaction.

Secondary Analysis A hierarchical procedure will be used to control type I error and handle multiple secondary endpoints analyses. If the primary endpoint analysis (ITT) is significant at $5 \%$ alpha level, key secondary efficacy endpoints will be tested sequentially in the order given in Table 2. In particular, LDL-C reduction at Week 24 will be analyzed on-treatment in the modified ITT population (i.e. patients with at least one baseline and at least one calculated LDL-C value available ontreatment between Week 4 and 24) if the primary analysis is significant in the ITT population.

Continuous secondary endpoints, except Lp(a) and triglycerides, will be analyzed using the same MMRM model as for the primary endpoint. $\mathrm{Lp}(\mathrm{a})$ and triglycerides (which have a non-Gaussian distribution), and the binary secondary endpoints (proportion of patients with LDL-C $<70 \mathrm{mg} / \mathrm{dL}$ and $<100 \mathrm{mg} / \mathrm{dL}$ ) will be analyzed using a multiple imputation approach for handling of missing values followed by robust regression [30] (for $\mathrm{Lp}(\mathrm{a})$ and triglycerides) or logistic regression (for binary endpoints).

\section{Safety Analysis}

AEs (including adjudicated CV events), laboratory parameters, and vital signs will be reported descriptively, based on the safety population (all randomized patients who received at least one dose or partial dose of study treatment). The safety analysis will focus on the TEAE period defined as the time from the first double-blind dose to the last double-blind dose of the investigational product +70 days ( 10 weeks).

Patients entering the open-label extension study will be followed up to their last visit in the double-blind treatment period and their TEAE period will be truncated at this visit.

Timing of Analysis The analysis will be conducted in two steps. The first step will be the main efficacy and safety analyses conducted as soon as all patients have been randomized and have at least all their data up to Week 52 collected and validated. In this step, the final analysis of the primary and secondary efficacy endpoints up to Week 52 will be performed as well as the safety analysis on all safety data collected and validated at the time of the first analysis.

Of note, the results of the first analysis will not be used to change the conduct of the ongoing study in any aspect. Since the analyses of primary and key secondary efficacy endpoints will be final at the time of first step analysis, the significance level for the study remains at 0.05 . The second step in the analysis will be conducted at the end of the study and will consist of the final analysis of Week 78 efficacy endpoints and the final safety analysis.

\section{Discussion}

A recent consensus statement has been issued urging a worldwide need to address the underdiagnosis and undertreatment of patients with heFH [4]. The prevalence of heFH comes from estimates based on a theoretical frequency of 1/500 in the general population, and recent numbers indicate that approximately 689,900 individuals in the USA and Canada, 527,500 in Europe and 100,000 in South Africa have heFH [4]. Furthermore, some studies have indicated the prevalence can range from $1 / 500$ to $1 / 200$, and based on extrapolations, it is estimated that there are between 14 and 34 million individuals with heFH worldwide [4]. In many countries, however, less than $1 \%$ of patients are diagnosed [4].

A diagnosis of heFH is typically made based on family history, clinical history of CHD, physical examination for xanthomas and corneal arcus, and very high levels of LDLC; it can also be made by genotyping [31]. However, despite the increased understanding of genetic causes and direct detection of mutations in the LDLR, APOB, PCSK9 and LDLRAP genes, the vast majority of diagnoses still rely on the more historical clinical criteria.

Although it has been demonstrated that statin therapy, with or without other LLT, can reduce the risk of CHD in patients with heFH [5-7], many patients with heFH still do not achieve desired LDL-C levels [8-12, 22, 24, 32, 33] and more 
intensive treatment options for these high-risk patients are required [4]. To help address this unmet need, new approaches to lowering LDL-C, including monoclonal antibodies to PCSK9 administered as subcutaneous injection, are currently in development.

In evaluating the efficacy and safety of such antibodies to PCSK9, a Phase 2, randomized controlled trial of alirocumab conducted in patients with heFH and LDL-C levels $>100 \mathrm{mg} /$ $\mathrm{dL}$ despite their current LLT of a statin, with or without ezetimibe, showed that alirocumab was well tolerated and also showed statistically significant reductions in LDL-C levels at all doses of alirocumab compared with placebo [24]. With the large reductions in LDL-C, up to $94 \%$ of patients achieved LDL-C levels $<100 \mathrm{mg} / \mathrm{dL}$ and up to $81 \%$ achieved levels $<70 \mathrm{mg} / \mathrm{dL}$ [24]. In another Phase 2, randomized controlled trial with evolocumab in heFH patients with LDL-C levels $>100 \mathrm{mg} / \mathrm{dL}$ despite receiving statin therapy, with or without ezetimibe, findings showed substantial reductions in LDL-C levels with evolocumab compared with placebo [22].

The ODYSSEY FH studies are Phase 3 studies aiming to further demonstrate the potential of alirocumab as an effective therapeutic option for patients with this severe disorder. The uptitration dosing strategy in FH I and FH II allows for flexibility and individualized therapy based on the degree of LDL-C reduction required to achieve the desired treatment response. In HIGH FH, where patients entered the study with LDL-C values $\geq 160 \mathrm{mg} / \mathrm{dL}$ despite receiving a maximally tolerated statin dose, with or without ezetimibe, it was deemed appropriate to initiate the higher, $150 \mathrm{mg}$ Q2W, dose of alirocumab. In addition, the option for patients to enter an open-label extension study at the end of the double-blind treatment period will allow for further assessment of the efficacy and safety of alirocumab.

Acknowledgments The ODYSSEY FH studies were supported by Sanofi and Regeneron Pharmaceuticals, Inc. The design and conduct of the study, as well as analysis of the study data and opinions, conclusions, and interpretation of the data, are the responsibility of the authors. Medical writing support in the preparation of this publication was provided by Betty Thompson, Ph.D., of Prime Medica and sponsored by Sanofi and Regeneron Pharmaceuticals, Inc. The authors were responsible for all content and editorial decisions and received no honoraria related to the development/presentation of this publication.

\section{Investigators FH I}

Canada: Robert DuFour (Montreal, Quebec); Jean Bergeron (SainteFoy, Quebec); Daniel Gaudet (Montreal, Quebec); Patrice Perron (Sherbrooke, Quebec); Lawrence Leiter (Toronto, Ontario)

United States: Thomas Barringer (Charlotte, NC); Robert Fishberg (Summit, NJ); Henry Ginsberg (New York, NY); Anne Goldberg (St Louis, MO); John Guyton (Durham, NC); Linda Hemphill (Boston, MA); John Homan (Newport Beach, CA); Patrick Moriarty (Kansas City, KS); Galal Salem (Bell Gardens, CA); Erich Schramm (Ponte Vedra, FL); Prediman Shah (Los Angeles, CA); Chad Wadell (Mission Viejo, CA); Ralph Wade (Bountiful, UT); Jonathan Purnell (Portland, OR); Robert
Weiss (Lewiston, ME); Marina Cuchel (Philadelphia, PA); Emanuel Shaoulian (Newport Beach, CA); Robert Greenfield (Fountain Valley, CA); Traci Turner (Cincinnati, OH); Alan Brown (Oakbrook Terrace, IL); Jeffrey Geohas (Evanston, IL); Frederick Dunn (Dallas, TX)

Austria: Rudolf Prager (Wien); Hermann Toplak (Graz); Evelyn Fliesser-Goerzer (St Stefan)

Czech Republic: Marketa Galovcova (Praha 4); Josef Machacek (Zlin); Jan Zeman (Praha 8); Stanislav Zemek (Uherské Hradiště)

Denmark: Erik Schmidt (Aalborg); Kristian Thomsen (Esbjerg); Pernille Correll (Roskilde)

France: Eric Bruckert (Paris); Michel Krempf (Saint-Herblain); Michel Farnier (Dijon); Gerald Luc (Lille)

Israel: Hofit Cohen (Tel-Hashomer); Dov Gavish (Holon); Osamah Hussein (Safed); Daniel Schurr (Jerusalem)

Netherlands: S.H.J. Donders (Groningen); G. Kees Hovingh (Amsterdam); P. Viergever (Den Helder); H.H. Vincent (Nieuwegein); A. Loualidi (Delfzijl); S.C.C. Hartong (Sliedrecht); A.A. Kroon (Maastricht); Jacqueline De Graaf (Nijmegen)

Norway: Anders Hovland (Bodo)

Russia: Evgenia Akatova (Moscow); Vadim Arkhipovsky (Arkhangelsk); Elena Demchenko (St. Petersburg); Victor Gurevich (St. Petersburg); Gadel Kamalov (Kazan); Anastasia Lebedeva (Moscow); Viacheslav Marasaev (Yaroslavl); Svetlana Mustafina (Novosibirsk); Andrey Susekov (Moscow)

Spain: Luis Alvarez-Sala (Madrid); Fernando Civeira (ZaragozaAragon); Jose Luis Diaz Diaz (La Coruna- Galicia); Nuria Plana Gil (Reus-Tarragona); Rodrigo Alonso (Madrid); Francisco Fuentes Jimenez (Cordoba); Xavier Pinto Sala (Barcelona); Emilio Ros (Barcelona); Jose Luis Mostaza Prieto (Madrid)

Sweden: A. Ohlsson-Önerud (Stockholm); Stefano Romeo (Goteborg)

United Kingdom: Graham Bayly (Bristol); Jacob George (Dundee); Basil Issa (Manchester); Gordon Ferns (Brighton)

South Africa: F.C.J. Bester (Bloemfontein); Dirk Blom (Cape Town); Lesley Burgess (Cape Town); Shaunagh Emanuel (Cape Town); Nyda Fourie (Bloemfontein); Maria Pretorius (Cape Town); Frederick Raal (Johannesburg); Prashilla Soma (Pretoria); Shirley Middlemost (Western Cape)

\section{FH II}

Czech Republic: Richard Ceska (Praha 2); Vladmir Blaha (Hradec Kralove); Jana Cepova (Praha 5); Hana Halamkova (Vyskov); Lucie Solcova (Trutnov); Jana Jirouskova (Praha 8).

Netherlands: G. Kees Hovingh (Amsterdam); Stan Peter Janssen (Utrecht); H.W.O. Roeters van Lennep (Goes); Roel P.T. Troquay (Venlo); B.E. Groenemeijer (Apeldoorn); J.W. Hans Louwerenburg (Enschede); Marcel A. van de Ree (Utrecht); Adriaan Kooy (Hoogeveen); Suat Simsek (Alkmaar); Ben P.M. Imholz (Waalwijk); P.W. Kamphuisen (Groningen); Castro Cabezas (Rotterdam); Dick Basart (Hoorn).

Norway: Gisle Langslet (Oslo); Eli Heggen (Oslo).

UK: See Kwok (Manchester); D.D.R. Nair (London); Alan Rees (Cardiff); R.D.G. Neely (Newcastle upon Tyne); Elizabeth Hughes (West Bromwich).

\section{HIGH FH}

Canada: Patrice Perron (Sherbrooke, Quebec); Jean Bergeron (Sainte-Foy, Quebec)

United States: Henry Ginsberg (New York, NY); John Guyton (Durham, NC); John Homan (Newport Beach, CA); Marina Cuchel (Philadelphia, PA); Rashmi Schramm (Ponte Vedra, FL); Robert Greenfield (Fountain Valley, CA); Traci Turner (Cincinnati, OH); Lisa Martin (Washington, DC); Frederick Dunn (Dallas, TX); Robert DeLuca (Miami, FL); Galal Salem (Bell Gardens, CA)

Netherlands: E.S.G. Stroes (Amsterdam); S.H.J. Donders (Groningen); Marcel A. van de Ree (Utrecht); J.P.R. Herrman (Amsterdam); M.V. Huisman (Leiden) 
Russia: Elena Demchenko (St. Petersburg); Anastasia Lebedeva (Moscow); Gadel Kamalov (Kazan); Vadim Arkhipovsky (Arkhangelsk); Viacheslav Marasaev (Yaroslavl); Evgenia Akatova (Moscow); Victor Gurevich (St. Petersburg); Andrey Susekov (Moscow)

South Africa: F.C.J. Bester (Bloemfontein); Frederick Raal (Johannesburg); Shirley Middlemost (Western Cape); Nyda Fourie (Bloemfontein); Maria Pretorius (Cape Town)

Steering Committee Chairman: Henry Ginsberg, MD (Irving Institute for Clinical and Translational Research, Columbia University New York, NY USA). Members: Jennifer G. Robinson, MD, MPH (The University of Iowa, Iowa City, IA USA); Daniel J. Rader, MD (Institute for Translational Medicine and Therapeutics, University of Pennsylvania School of Medicine Philadelphia, PA USA); Christopher P. Cannon, MD (Senior Investigator, TIMI Study Group; Professor of Medicine, Harvard Medical School Cardiovascular Division, Brigham and Women's Hospital, Boston, MA USA); Helen Colhoun, MD, MFPHM (Clinical Centre, University of Dundee, Ninewells Hospital \& Medical School, Dundee, UK); John J.P. Kastelein, MD (Academic Medical Center University of Amsterdam, Amsterdam, The Netherlands); Michel Farnier, MD (Point Médical, Département d'Endocrinologie et de Lipidologie, Dijon, France).

Data Monitoring Committee DMC Chairman: Anders Olsson, MD (Bromma, Sweden). Members: David Waters, MD (Division of Cardiology, San Francisco General Hospital, San Francisco, CA USA); Dominique Larrey, MD (Hôpital Saint-Eloi Service d'hépato-gastroentérologie, Montpellier, France); Robert S Rosenson, MD (Director, Cardiometabolic Disorders, Mount Sinai Heart; Professor of Medicine, Mount Sinai School of Medicine, New York, NY USA); Peter A. Patriarca, MD (Biologics Consulting Group, Inc., Alexandria, VA USA); Geert Molenberghs, Biostatistician (Center for Statistics (CenStat) Universiteit Hasselt Agoralaan 1, Diepenbeek, Belgium).

Clinical Events Committee (Reviewers), Duke Clinical Research Institute, Durham, NC USA Pierluigi Tricoci, CEC Principal Investigator, Cardiology; Kenneth W Mahaffey, CEC Director, Cardiology; Renato D Lopes, Cardiology; Bimal R Shah, Cardiology; Rajendra H Metha, Cardiology; Matthew T Roe, Cardiology; Zubin Eapen, Cardiology; Luciana Armaganijan, Cardiology; Adriana Bertolami, Cardiology; Sergio Leonardi, Cardiology; Bradley J. Kolls, Neurology; J. Dedrick Jordan, Neurology; Grégory Ducrocq, Cardiology; Etienne Puymirat, Cardiology; Robin Mathews, Cardiology.

Disclosures Dr. Kastelein is a consultant to and receives honoraria from: Regeneron, Sanofi, Eli Lilly, Pfizer, Amgen, Isis, Genzyme, Aegerion and Esperion. He is a recipient of the Lifetime Achievement Award of the Dutch Heart Foundation (2010T082).

Dr. Robinson is employed by a University that has received research funds from Amarin, Amgen, AstraZeneca, Daiichi-Sankyo, Esperion, Genetech/Hoffman La Roche, Glaxo-Smith Kline, Merck, Regeneron/ Sanofi, Zinfandel/Takeda and is a consultant for Amgen, Pfizer, Sanofi and Regeneron.

Dr. Farnier reports having received grants, consulting fees and/or honoraria and delivering lectures for Abbott, Amgen, BoehringerIngelheim, Genzyme, Kowa, Merck and Co, Novartis, Pfizer, Recordati, Roche, Sanofi-Aventis, and SMB.

Dr. Langslet has received advisory board fees from Sanofi-Aventis and Janssen Pharmaceuticals.

Dr. Krempf has received grants, consulting fees and/or honoraria and delivering lectures for Abbott, Amgen, Astra Zeneca, BMS, Merck and Co, Novartis, Pfizer, Roche, Sanofi-Aventis.

Christelle Lorenzato and Marie T. Baccara-Dinet are employees of Sanofi.

Daniel A. Gipe is an employee of Regeneron.
Open Access This article is distributed under the terms of the Creative Commons Attribution License which permits any use, distribution, and reproduction in any medium, provided the original author(s) and the source are credited.

\section{References}

1. Goldstein JL, Hobbs HH, Brown MS. Familial hypercholesterolemia. In: Scriver CR, Sly WS, Childs B, et al., editors. The metabolic and molecular bases of inherited disease. 8th ed. New York: McGraw-Hill; 2001. p. 2863-913.

2. NICE clinical guidelines 71. Identification and management of familial hypercholesterolemia. Issue date: August 2008. Available from: URL: http://www.nice.org.uk/nicemedia/live/12048/41697/ 41697.pdf. Accessed Mar 2013.

3. Stone NJ, Levy RI, Fredrickson DS, et al. Coronary artery disease in 116 kindred with familial type II hyperlipoproteinemia. Circulation. 1974;49:476-88.

4. Nordestgaard BG, Chapman MJ, Humphries SE, for the European Atherosclerosis Society Consensus Panel, et al. Familial hypercholesterolaemia is underdiagnosed and undertreated in the general population: guidance for clinicians to prevent coronary heart disease: Consensus Statement of the European Atherosclerosis Society. Eur Heart J. 2013;34:3478-90a.

5. Neil A, Cooper J, Betteridge J, et al. Reductions in all-cause, cancer, and coronary mortality in statin-treated patients with heterozygous familial hypercholesterolaemia: a prospective registry study. Eur Heart J. 2008;29:2625-33.

6. Harada-Shiba M, Sugisawa T, Makino H, et al. Impact of statin treatment on the clinical fate of heterozygous familial hypercholesteremia. J Atheroscler Thromb. 2010;17:667-74.

7. Versmissen J, Oosterveer DM, Yazdanpanah M, et al. Efficacy of statins in familial hypercholesterolaemia: a long term cohort study. BMJ. 2008;337:a2423.

8. Huijgen R, Kindt I, Verhoeven S, et al. Two years after molecular diagnosis of familial hypercholesterolemia: majority on cholesterollowering treatment but a minority reaches treatment goal. PLoS ONE. 2010;5:e9220.

9. Gitt AK, Jünger C, Smolka W, Bestehorn K. Prevalence and overlap of different lipidabnormalities in statin-treated patients at high cardiovascular risk in clinical practice in Germany. Clin Res Cardiol. 2010;99:723-33.

10. Goldberg AC, Hopkins PN, Toth PP, et al. Familial hypercholesterolemia: screening, diagnosis and management of pediatric and adult patients: clinical guidance from the National Lipid Association Expert Panel on Familial Hypercholesterolemia. J Clin Lipidol. 2011;5(3, Supplement 1):S1-8.

11. Pijlman AH, Huijgen R, Verhagen SN, et al. Evaluation of cholesterol lowering treatments of patients with familial hypercholesterolemia: a large cross-sectional study in The Netherlands. Atherosclerosis. 2010;209:189-94.

12. Stein EA, Strutt K, Southworth H, HeFH study group, et al. Comparison of rosuvastatin versus atorvastatin in patients with heterozygous familial hypercholesterolemia. Am J Cardiol. 2003;92:1287-93.

13. Stone N, Robinson J, Lichtenstein A, et al. ACC/AHA guideline on the treatment of blood cholesterol to reduce atherosclerotic cardiovascular risk in adults. Circulation. 2013.

14. Abifadel M, Varret M, Rabes J-P, et al. Mutations in PCSK9 cause autosomal dominant hypercholesterolemia. Nat Genet. 2003;34: 154-6.

15. Lagace TA, Curtis DE, Garuti R, et al. Secreted PCSK9 decreases the number of LDL receptors in hepatocytes and in livers of parabiotic mice. J Clin Invest. 2006;116:2995-3005. 
16. Maxwell KN, Fisher EA, Breslow JL. Overexpression of PCSK9 accelerates the degradation of the LDLR in a post-endoplasmic reticulum compartment. Proc Natl Acad Sci U S A. 2005;102: 2069-74.

17. Mayne J, Dewpura T, Raymond A, et al. Novel loss-of-function PCSK9 variant is associated with low plasma LDL cholesterol in a French-Canadian family and with impaired processing and secretion in cell culture. Clin Chem. 2011;57:1415-23.

18. Welder G, Zineh I, Pacanowski MA, et al. High-dose atorvastatin causes a rapid sustained increase in human serum PCSK9 and disrupts its correlation with LDL cholesterol. J Lipid Res. 2010;51: 2714-21.

19. Giugliano RP, Desai NR, Kohli P, et al. Efficacy, safety, and tolerability of a monoclonal antibody to proprotein convertase subtilisin/ kexin type 9 in combination with a statin in patients with hypercholesterolaemia (LAPLACE-TIMI 57): a randomised, placebocontrolled, dose-ranging, phase 2 study. Lancet. 2012;380:2007-17.

20. Koren MJ, Scott R, Kim JB, et al. Efficacy, safety, and tolerability of a monoclonal antibody to proprotein convertase subtilisin/kexin type 9 as monotherapy in patients with hypercholesterolaemia (MENDEL): a randomised, double-blind, placebo-controlled, phase 2 study. Lancet. 2012;380:1995-2006.

21. McKenney JM, Koren MJ, Kereiakes DJ, et al. Safety and efficacy of a monoclonal antibody to proprotein convertase subtilisin/kexin type 9 serine protease, SAR236553/RENGN727, in patients with primary hypercholesterolemia receiving ongoing stable atorvastatin therapy. J Am Coll Cardiol. 2012;59:2344-53.

22. Raal F, Scott R, Somaratne R, et al. Low-density lipoprotein cholesterol-lowering effects of AMG-145, a monoclonal antibody to proprotein convertase subtilisin/kexin type 9 serine protease in patients with heterozygous familial hypercholesterolemia: the reduction in LDL-C with PCSK9 inhibition in heterozygous familial hypercholesterolemia disorder (RUTHERFORD) randomized trial. Circulation. 2012;126:2408-17.

23. Roth EM, McKenney JM, Hanotin C, et al. Atorvastatin with or without an antibody to PCSK9 in primary hypercholesterolemia. N Engl J Med. 2012;367:1891-900.
24. Stein EA, Gipe D, Bergeron J, et al. Effect of a monoclonal antibody to PCSK9, REGN727/SAR236553, to reduce low-density lipoprotein cholesterol in patients with heterozygous familial hypercholesterolaemia on stable statin dose with or without ezetimibe therapy: a phase 2 randomised controlled trial. Lancet. 2012;380:29-36.

25. Sullivan D, Olsson AG, Scott R, et al. Effect of a monoclonal antibody to PCSK9 on low-density lipoprotein cholesterol levels in statin-intolerant patients. The GAUSS randomized trial. JAMA. 2012;308:1545-54

26. Scientific Steering Committee on behalf of the Simon Register Group. Risk of fatal coronary heart disease in familial hypercholesterolaemia. BMJ. 1991;303:893-6.

27. World Health Organization. Familial Hypercholesterolaemia (FH): report of a second WHO consultation. Geneva: World Health Organization; 1999.

28. Siddiqui O, Hung HM, O’Neill R. MMRM vs. LOCF: a comprehensive comparison based on simulation study and 25 NDA datasets. $\mathrm{J}$ Biopharm Stat. 2009;19:227-46.

29. National Research Council. The prevention and treatment of missing data in clinical trials. Panel on Handling Missing Data in Clinical Trials. Committee on National Statistics, Division of Behavioral and Social Sciences and Education. Washington, DC: The National Academies Press; 2010.

30. Mehrotra DV, Li X, Liu J, Lu K. Analysis of longitudinal clinical trials with missing data using multiple imputation in conjunction with robust regression. Biometrics. 2012;68:1250-9.

31. Civeira F. Guidelines for the diagnosis and management of heterozygous familial hypercholesterolemia. Atherosclerosis. 2004;173: $55-68$.

32. Stein E, Stender S, Mata P, Ezetimibe Study Group, et al. Achieving lipoprotein goals in patients at high risk with severe hypercholesterolemia: efficacy and safety of ezetimibe co-administered with atorvastatin. Am Heart J. 2004;148:447-55.

33. Stein EA, Ose L, Retterstol K, et al. Further reduction of low-density lipoprotein cholesterol and C-reactive protein with the addition of ezetimibe to maximum-dose rosuvastatin in patients with severe hypercholesterolemia. J Clin Lipidol. 2007;1:280-6. 